\title{
Simulation and validation of temperature-dependent ferroelectric properties of multifunctional BCZT and KNBNNO ceramics
}

Yang Bai ${ }^{*}$, Benjamin Ducharne ${ }^{2 *}$, Heli Jantunen ${ }^{1}$ and Jari Juuti ${ }^{1}$

${ }^{1}$ Microelectronics Research Unit, Faculty of Information Technology and Electrical Engineering, University of Oulu, FI-90014 Oulu, Finland

${ }^{2}$ Laboratoire de Génie Electrique et Ferroélectricité, INSA Lyon, 69621 Villeurbanne, France

*Correspondence: yang.bai@oulu.fi; benjamin.ducharne@insa-lyon.fr

\begin{abstract}
The properties of perovskite-structured piezoelectric, pyroelectric and photovoltaic materials are largely dependent on their ferroelectric behaviors, e.g. spontaneous and remanent polarizations. Being able to simulate and predict the ferroelectric properties enables better design and optimization of these materials. In this paper, a ferroelectric model is developed from the original ferromagnetic Jiles-Atherton model, with the incorporation of dynamic and temperature contributions. The model is used to compute the ferroelectric hysteresis loops of two ferroelectric materials at various temperatures - $\left(\mathrm{Ba}_{0.85} \mathrm{Ca}_{0.15}\right)\left(\mathrm{Zr}_{0.1} \mathrm{Ti}_{0.9}\right) \mathrm{O}_{3}(\mathrm{BCZT})$ exhibiting excellent piezoelectric and pyroelectric properties and $\left(\mathrm{K}_{0.485} \mathrm{Na}_{0.485} \mathrm{Ba}_{0.03}\right)\left(\mathrm{Nb}_{0.985} \mathrm{Ni}_{0.015}\right) \mathrm{O}_{2.9925}$ which is very recently discovered to obtain piezoelectric, pyroelectric and photovoltaic properties simultaneously. Good agreement between the simulation and measurement is achieved, with a $<5 \%$ difference between the simulated and measured polarization values in the ferroelectric hysteresis loops (P-E loops). By predicting their ferroelectric behaviour, the model will stimulate the development of high-performance ferroelectric materials for applications such as sensing and energy harvesting.
\end{abstract}




\section{Introduction}

Piezoelectric and pyroelectric materials play an important role in smart systems by being the core functional components of kinetic and thermal sensors, actuators, transducers and energy harvesters [1-4]. High-performance piezoelectric and pyroelectric materials are perovskite-structured, e.g. $\mathrm{Pb}(\mathrm{Zr}, \mathrm{Ti}) \mathrm{O}_{3}(\mathrm{PZT}), \mathrm{BaTiO}_{3}(\mathrm{BTO}),\left(\mathrm{K}_{0.5} \mathrm{Na}_{0.5}\right) \mathrm{NbO}_{3}(\mathrm{KNN})[1,5]$. These perovskites have large spontaneous polarizations, thus giving them the potential to exhibit strong piezoelectric and pyroelectric responses. For instance, for the same material, a larger remanent polarization usually induces a larger piezoelectric charge coefficient and a larger electromechanical coupling coefficient [3]. The alignment of domains also influences the macroscopic permittivity [3]. These parameters are all closely related to the performance of different piezoelectric devices. Similarly, in order to extract the maximum possible electric charges due to temperature fluctuations and thus obtain the optimum pyroelectric effect, a larger variation of the remanent polarization at different temperatures is usually preferred $[2,6]$.

Meanwhile, the performance of some multi-functional materials based on perovskite structures is also partially dependent on the polarization, i.e. ferroelectric properties. For instance, multi-functional perovskites such as $\left(\mathrm{KNbO}_{3}\right)_{1-\mathrm{x}-}\left[\mathrm{Ba}\left(\mathrm{Ni}_{0.5} \mathrm{Nb}_{0.5}\right) \mathrm{O}_{3-\beta}\right]_{\mathrm{x}} \quad(\mathrm{KBNNO})$ and $\left[\left(\mathrm{K}_{0.5} \mathrm{Na}_{0.5}\right) \mathrm{NbO}_{3}\right]_{1-\mathrm{x}^{-}}$ $\left[\mathrm{Ba}\left(\mathrm{Ni}_{0.5} \mathrm{Nb}_{0.5}\right) \mathrm{O}_{3-\beta}\right]_{\mathrm{x}}(\mathrm{KNBNNO})$ have very recently been found to be attractive because of their coexhibition of a narrow band gap and strong ferroelectricity $[7,8]$. This makes them useful in multisource energy harvesters and sensors, simultaneously responding to inputs of kinetic, thermal and solar energy. In these materials, the remanent polarization is also crucial to maintain a good level of piezoelectric and pyroelectric properties despite a reasonable compromise to narrow band gaps [7-9]. Table 1 gives an overview of the relationship between remanent polarization and other properties for the KNBNNO. In the table, different KNBNNO compositions are sintered at different temperatures, causing the remanent polarization varying largely. It is clear that while the band gaps are maintained at the same level for those compositions with $\mathrm{x}=0.02-0.08$, the remanent polarization obviously 
influences the piezoelectric, pyroelectric and dielectric properties. The piezoelectric and pyroelectric coefficients as well as the relative permittivity were measured after the samples were poled under the same conditions, i.e. at $90 \mathrm{kV} / \mathrm{cm}$ electric field in silicone oil at room temperature [8]. Furthermore, being simultaneously photo-ferroelectric, the photovoltaic performance of the perovskites is also closely related to spontaneous polarizations [10]

Table 1. Summary of the material and functional properties of the KNBNNO. Reproduced with permission [8]. Copyright Wiley, 2017.

\begin{tabular}{|c|c|c|c|c|c|c|}
\hline Composition & $\begin{array}{c}\text { Sintering } \\
\text { temperature } \\
\left({ }^{\circ} \mathrm{C}\right)\end{array}$ & $\begin{array}{c}\text { Remanent } \\
\text { polarization }\left(P_{r}\right) \\
\text { at } 1 \mathrm{~Hz} \\
{\left[\mu \mathrm{C} / \mathrm{cm}^{2}\right]}\end{array}$ & $\begin{array}{c}\text { Bandgap } \\
{[\mathrm{eV}]}\end{array}$ & $\begin{array}{c}\text { Piezoelectric } \\
\text { coefficient }\left(d_{33}\right) \\
{[\mathrm{pm} / \mathrm{V}]}\end{array}$ & $\begin{array}{c}\text { Pyroelectric } \\
\text { coefficient }(\gamma) \\
{\left[\mu \mathrm{C} / \mathrm{m}^{2} \mathrm{~K}\right]}\end{array}$ & $\begin{array}{c}\text { Relative } \\
\text { permittivity }\left(\varepsilon_{r}\right) \\
\text { at } 1 \mathrm{kHz}\end{array}$ \\
\hline$\left(\mathrm{K}_{0.5} \mathrm{Na}_{0.5}\right) \mathrm{NbO}_{3}$ & 1135 & 20 & $>4$ & 100 & 140 & 472 \\
\hline \multirow{4}{*}{$\begin{array}{l}{\left[\left(\mathrm{K}_{0.5} \mathrm{Na}_{0.5}\right) \mathrm{NbO}_{3}\right]_{0.98^{-}}} \\
{\left[\mathrm{Ba}\left(\mathrm{Ni}_{0.5} \mathrm{Nb}_{0.5}\right) \mathrm{O}_{3-\beta}\right]_{0.02}}\end{array}$} & 1165 & 11.3 & 1.59 & 100 & 128 & 934 \\
\hline & 1175 & 6.5 & 1.63 & 66 & 77 & 863 \\
\hline & 1185 & 5.6 & 1.58 & 57 & 31 & 812 \\
\hline & 1195 & 7.2 & 1.58 & 41 & 15 & 796 \\
\hline \multirow{3}{*}{$\begin{array}{l}{\left[\left(\mathrm{K}_{0.5} \mathrm{Na}_{0.5}\right) \mathrm{NbO}_{3}\right]_{0.96^{-}}} \\
{\left[\mathrm{Ba}\left(\mathrm{Ni}_{0.5} \mathrm{Nb}_{0.5}\right) \mathrm{O}_{3-\beta}\right]_{0.04}}\end{array}$} & 1175 & 0.9 & 1.55 & $<10$ & 9.3 & 660 \\
\hline & 1185 & 0.9 & 1.50 & $<10$ & 6.2 & 659 \\
\hline & 1195 & 4.3 & 1.50 & $<10$ & 6.0 & 684 \\
\hline$\left[\left(\mathrm{K}_{0.5} \mathrm{Na}_{0.5}\right) \mathrm{NbO}_{3}\right]_{0.94^{-}}$ & 1185 & 0.6 & 1.58 & - & 3.1 & 639 \\
\hline$\left[\mathrm{Ba}\left(\mathrm{Ni}_{0.5} \mathrm{Nb}_{0.5}\right) \mathrm{O}_{3-\beta}\right]_{0.06}$ & 1195 & 0.6 & 1.56 & - & 3.9 & 636 \\
\hline $\begin{array}{c}{\left[\left(\mathrm{K}_{0.5} \mathrm{Na}_{0.5}\right) \mathrm{NbO}_{3}\right]_{0.92^{-}}} \\
{\left[\mathrm{Ba}\left(\mathrm{Ni}_{0.5} \mathrm{Nb}_{0.5}\right) \mathrm{O}_{3-\beta}\right]_{0.08}}\end{array}$ & 1195 & 0.6 & 1.56 & - & 3.1 & 668 \\
\hline $\begin{array}{l}{\left[\left(\mathrm{K}_{0.5} \mathrm{Na}_{0.5}\right) \mathrm{NbO}_{3}\right]_{0.9^{-}}} \\
{\left[\mathrm{Ba}\left(\mathrm{Ni}_{0.5} \mathrm{Nb}_{0.5}\right) \mathrm{O}_{3-\beta}\right]_{0.1}}\end{array}$ & 1195 & $\approx 0$ & 1.16 & - & n.a. & 632 \\
\hline
\end{tabular}

Therefore, being able to predict the ferroelectric properties enables better design and optimization of these materials. This becomes very beneficial if the materials are to be operated at extremely high or low temperature in order to predict how they will behave in these environments. However, at the current time, ferroelectric properties have not been accurately simulated for all materials, especially when considering temperature variation. In this paper, a developed model for the computation of ferroelectric hysteresis loops is presented, enabling accurate prediction of the ferroelectric properties such as saturation and remanent polarizations at different temperatures. A lead-free piezoelectric 
composition - $\left(\mathrm{Ba}_{0.85} \mathrm{Ca}_{0.15}\right)\left(\mathrm{Zr}_{0.1} \mathrm{Ti}_{0.9}\right) \mathrm{O}_{3}(\mathrm{BCZT})$ - which exhibits excellent piezoelectric and pyroelectric properties, as well as the very recently discovered photo-ferroelectric $\left(\mathrm{K}_{0.485} \mathrm{Na}{ }_{0.485} \mathrm{Ba}_{0.03}\right)\left(\mathrm{Nb}_{0.985} \mathrm{Ni}_{0.015}\right) \mathrm{O}_{2.9925}$, are used to validate the model. The reasons of choosing these two materials are as follows.

(1) BCZT and $\mathrm{KNN}$ are the most promising lead-free candidates to replace the toxic and $\mathrm{Pb}$ containing but widely used PZT for sensing/transducing and actuating applications at room and elevated temperatures, respectively $[5,11]$.

(2) As this paper introduces the temperature dependence, BCZT is an ideal candidate to validate the model because of the large variation of the P-E loops in a relatively narrow temperature range (within $\left.100{ }^{\circ} \mathrm{C}\right)[11]$.

(3) KNBNNO is developed from KNN. Not only its piezoelectric but also photovoltaic properties are found to be dependent on the ferroelectric properties [12]. Therefore, validating the model using KNBNNO may support relevant investigations on the multi-functional working mechanism.

Good agreement between the calculated and measured results is obtained. This will stimulate the progress of the development of models for high-performance ferroelectric materials used, for example, in energy harvesting, by predicting their ferroelectric behavior.

\section{Model}

\subsection{Quasi-static contribution - the Jiles-Atherton model}

Below a threshold frequency (in the decreasing direction), the cumulative periodic value of the dielectric losses becomes frequency-independent. This behavior is known as a quasi-static state. Its contribution is observable by plotting the spontaneous average dielectric polarization $(P)$ versus the excitation electric field $(E)$ in the very low frequency limit ( $<<1 \mathrm{~Hz}$ in a typical soft PZT composition). In this study, a scalar consideration of both $P$ and $E$ is relevant as both fields are collinear. Different 
approaches are available in the literature for the simulation of the scalar quasi-static hysteresis behavior. Among these, the Preisach and Jiles-Atherton (J-A) models in their original versions are the most popular for obtaining the scalar quasi-static hysteresis behavior of ferroelectric materials. Both models were originally used to describe hysteresis phenomena in magnetic materials [13-18]. Smith et al. proposed an adapted version of the ferromagnetic J-A model to cover ferroelectric materials [19]. This model is based on the consideration of two contributions - domain wall bending and domain wall translations. It should be noted that the J-A model in its ferroelectric version exhibits some particularly interesting features as follows.

- It requires only a small memory storage, where the evolution history information is limited to a single time step before $P$.

- The initial ferroelectric version of the Jiles-Atherton model relies on only five parameters. The effect of each parameter is available in reference [19].

- The five parameters are related to the microstructural property, which allows a physical interpretation of the J-A model.

- As is the case for the Preisach model, the J-A model is easily reversible and relatively easy to switch from $E$ to $P$ as the input for the quasi-static hysteresis model.

In the first version of the scalar J-A model for ferroelectric materials, $P$ is decomposed into its reversible $\left(P_{\text {rev }}\right)$ and irreversible $\left(P_{i r r}\right)$ components. These two contributions are linked according to Equation 1, in which $c$ is the polarization reversibility.

$P_{\text {rev }}=c\left(P_{a n}-P_{\text {irr }}\right)$

The anhysteretic polarization $\left(P_{a n}\right)$ comes from the Langevin function as shown in Equation 2, in which $P_{s}$ is the saturation polarization measured in the hysteresis loop and $a$ is the density of domain walls in the dielectric material.

$P_{a n}=P_{S} \cdot\left[\operatorname{coth}\left(\frac{E_{e}}{a}\right)-\frac{a}{E_{e}}\right]$ 
Alternatively, $P_{a n}$ can be derived from the Ising spin model as shown in Equation 3, where $E_{e}$ is the effective electric field defined by Equation 4. In Equation 4, $\alpha$ is the inter-domain coupling in the dielectric material and $E$ is the applied electric field.

$P_{a n}=P_{s} \cdot \tanh \left(\frac{E_{e}}{a}\right)$

$E_{e}=E+\alpha P$

In this study, better results are obtained by modifying Equation 3 to Equation 5, considering a second term yielding an equivalent parallel capacitance which creates a small positive drift on the hysteresis cycle as saturation levels are reached. In Equation 5, $\varepsilon_{0}$ and $\varepsilon_{r}$ are the permittivity of vacuum space and the relative permittivity the material, respectively. As the $\varepsilon_{r}$ in this case is used for the simulation of the anhysteretic behavior, it is treated as a constant and is independent on the polarization state.

$P_{a n}=P_{s} \cdot \tanh \left(\frac{E_{e}}{a}\right)+\varepsilon_{0} \cdot \varepsilon_{r} \cdot\left(E_{e}\right)$

$P_{i r r}$ is defined in Equation 6, in which $k$ is the average energy required to break the pinning site in the dielectric material (pinning energy factor). A larger $k$ value produces wider hysteresis curve and narrower minor loops [19]. $\delta$ is a directional parameter taking on the value of either +1 (if $d E(t) / d t>$ 0 ) or -1 (if $d E(t) / d t<0$ ).

$\frac{d P_{i r r}}{d E_{e}}=\frac{P_{a n}-P_{i r r}}{k \delta}$

Combining the equations described above, the main equation of the original J-A model can be written as Equation 7.

$\frac{d P}{d E}=\frac{(1-c) \cdot \frac{d P_{i r r}}{d E_{e}}+c \cdot \frac{d P_{a n}}{d E_{e}}}{1-\alpha(1-c) \cdot \frac{d P_{i r r}}{d E_{e}}-\alpha c \cdot \frac{d P_{a n}}{d E_{e}}}$

This differential equation allows the calculation of $P$ with respect to variations in $E$. Although the original $\mathrm{J}$-A model is a quasi-static hysteresis model, by adding a dynamic contribution it can be extended to the simulation of the hysteresis behavior at higher frequency values. In the method used 
in this study, in order to extend the J-A model for the consideration of dynamic hysteresis cycles, $P$ is known before $E$. An inverse model for the ferromagnetic materials with $P$ as the model excitation has been described in detail [20]. The physical principles of the models are similar, and the main equation of this inverse model for ferroelectric materials then becomes Equation 8 .

$\frac{d P}{d E}=\frac{(1-c) \cdot \frac{d P_{i r r}}{d P_{e}}+\frac{c}{\varepsilon_{0}} \cdot \frac{d P_{a n}}{d E_{e}}}{1+\varepsilon_{0}(1-\alpha)(1-c) \cdot \frac{d P_{i r r}}{d P_{e}}-c(1-\alpha) \cdot \frac{d P_{a n}}{d E_{e}}}$

$P_{i r r}$ and the effective poling polarization $\left(P_{e}\right)$ can be defined in Equations 9 and 10.

$\frac{d P_{i r r}}{d P_{e}}=\frac{P_{a n}-P_{i r r}}{\varepsilon_{0} k \delta}$

$P_{e}=\varepsilon_{0} \cdot E_{e}$

\subsection{Dynamic contribution - Extension of the J-A models to dynamic behavior}

At low frequencies, scalar quasi-static lump hysteresis models provide accurate results for the evolution of $P$ versus $E$. Unfortunately, for this relatively simple mode, huge differences appear as soon as the quasi-static external condition expires, i.e. when the frequency increases. It has been demonstrated that small improvements can be obtained by adding a simple dynamic contribution to this lump model, i.e. a damping term $(\rho)$ proportional to the time derivative of $P$ shown as follows $[21]$.

$\rho \cdot \frac{d P}{d t}$

This term is homogeneous to an equivalent excitation field $E$. Much better results on a large frequency bandwidth have been obtained by replacing the time derivative of $P$ by a fractional one as shown below [21-25].

$\rho \cdot \frac{d^{n} P}{d t^{n}}$ 
The Riemman Liouville form for $\mathrm{n} \in[0,1]$ described in Equation 11 was used in this study for the fractional derivate numerical solution [26-29].

$\frac{d^{n} f(t)}{d t^{n}}=D_{t}^{n} f(t)=\frac{1}{\Gamma(1-n)} \cdot \frac{d}{d t} \int_{-\infty}^{t}(t-\tau)^{-n} f(\tau) d \tau$

In Equation 11, $\Gamma$ is the Euler gamma function and $n$ is the order of the fractional derivation. Equation 12 gives the latest version of the J-A model including both quasi-static and dynamic contributions.

$\rho \cdot \frac{d^{n} P(t)}{d t^{n}}=E_{\text {dynamic }}(t)-J-A_{\text {static }}^{-1}(P(t))$

The block diagram of the dynamic ferroelectric hysteresis model in this study is shown in Figure 1.

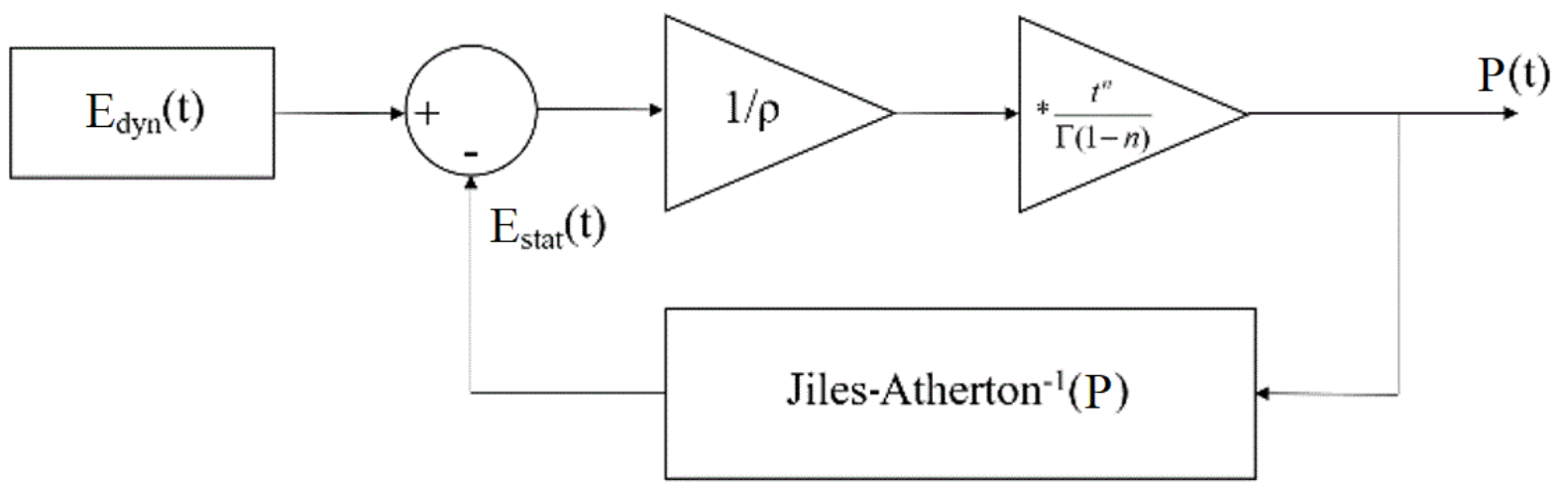

Figure 1. Block diagram of the dynamic ferroelectric hysteresis model.

\subsection{Temperature dependence - Extension of the ferroelectric J-A models to temperature dependence}

A temperature-dependent extended version of the J-A model has been proposed [30]. This modification in the original version of the J-A model promotes an understanding of the behavior of the magnetization process in ferromagnetic materials at different temperatures. In this study, thermal effects are incorporated through temperature-dependent hysteresis parameters of the original J-A model, with a material dependent critical exponent $(\beta)$ (according to the mean field theory [31]) and Curie temperature $\left(T_{c}\right)$ as additional parameters. For the ferroelectric materials, a similar modification 
to that for ferromagnetic materials can be applied for parameters $P_{s}, k, a, \alpha$ and $c$, as shown in Equations 13-17, respectively. In the equations, $T$ is temperature, and $P_{s}(0), k(0), a(0), \alpha(0)$ and $c(0)$ are the corresponding parameters at $0 \mathrm{~K}$.

As in the case for ferromagnetic materials, the temperature dependence of $P_{s}$ can be expressed for ferroelectric materials using the Weiss theory [20].

$P_{S}(T)=P_{S}(0) \cdot\left(1-\frac{T}{T_{c}}\right)^{\beta}$

For ferroelectric materials, $k$ also exhibits an exponentially dependence of the temperature.

$k(T)=k(0) \cdot e^{\left(\frac{-2 T}{\beta T_{c}}\right)}$

The parameter $a$ was previously treated as a constant by some researchers as it was claimed that the domain density variations were negligible [30]. However, such an assumption leads to incorrect simulation results in this study for ferroelectric materials. Better agreements are achieved by modifying parameter $a$ according to the modification law used for spontaneous polarization.

$a(T)=a(0) \cdot\left(1-\frac{T}{T_{C}}\right)^{\beta}$

$\alpha(T)=\alpha(0) \cdot\left(1-\frac{T}{T_{C}}\right)^{\beta}$

$c(T)=c(0) \cdot\left(1-\frac{T}{T_{C}}\right)^{\beta}$

In addition, $\varepsilon_{r}$ (in Equation 5) is considered to be independent of temperature, with good agreements still obtained between the simulation and measured results. It should also be noted that the power parameter shown in Equations 16 and 17 is $+\beta$ instead of $-\beta$ as specified [30].

The general identification procedure of the quasi-static and dynamic contribution parameters is not discussed in this paper, as there are existing references for the J-A model parameter identification [32, $33]$ and for the dynamic contributions [22, 23, 25]. Concerning the frequency dependence parameters, $T_{c}$ can be measured and $\beta$ can be obtained by the fitting identification with experimental results. 
The consideration of the temperature dependence in the simulation introduced two additional parameters to the five parameters in the initial ferroelectric version of the Jiles-Atherton model as described above. Both the additional parameters can be deduced from experimental results. The values of the parameters used for BCZT and KNBNNO in the validation presented below are given in Table 2.

Table 2. Values of the parameters used for BCZT and KNBNNO in the validation.

\begin{tabular}{cccccccc}
\hline & \multicolumn{4}{c}{ Ferroelectric model parameters } & \multicolumn{2}{c}{ Temperature dependence parameters } \\
\cline { 2 - 8 } & $\alpha(\mathrm{Vm} / \mathrm{C})$ & $a$ & $k\left(\mathrm{C} / \mathrm{m}^{2}\right)$ & $c$ & $P_{s}\left(\mu \mathrm{C} / \mathrm{cm}^{2}\right)$ & $\beta$ & $T_{c}\left({ }^{\circ} \mathrm{C}\right)$ \\
\hline BCZT & $2.5 \times 10^{-5}$ & $4.6 \times 10^{5}$ & $1.0 \times 10^{6}$ & 0.3 & $15.50 \times 10^{9}$ & 0.45 & 130 \\
KNBNNO & $3.0 \times 10^{-5}$ & $1.6 \times 10^{6}$ & $2.0 \times 10^{6}$ & 0.001 & $17.75 \times 10^{9}$ & - & - \\
\hline
\end{tabular}

\section{Experimental}

The $\left(\mathrm{Ba}_{0.85} \mathrm{Ca}_{0.15}\right)\left(\mathrm{Zr}_{0.1} \mathrm{Ti}_{0.9}\right) \mathrm{O}_{3}(\mathrm{BCZT})$ ceramic powder was synthesized through a conventional mixed oxide solid state reaction. $\mathrm{BaZrO}_{3}$ powder was fabricated first as a precursor. Stoichiometric amounts of $\mathrm{BaCO}_{3}$ (99\%, Alfa Aesar, USA) and $\mathrm{ZrO}_{2}$ (99\%, Sigma-Aldrich, USA) raw powders were mixed and milled in a $\mathrm{ZrO}_{2}$ jar with ethanol, using a planetary ball mill (Pulverisette 6, Fritsch, Idar-Oberstein, Germany) and with $3 \mathrm{~mm} \mathrm{ZrO}_{2}$ balls for 6 hours, followed by drying at $80{ }^{\circ} \mathrm{C}$. The mixture was calcined at $1400{ }^{\circ} \mathrm{C}$ for 10 hours with a $5{ }^{\circ} \mathrm{C} \mathrm{min}^{-1}$ ramp rate in a muffle furnace (Nabertherm, Germany). Subsequently, treating the synthesized $\mathrm{BaZrO}_{3}$ as one of the starting materials, stoichiometric amounts of $\mathrm{BaZrO}_{3}, \mathrm{CaCO}_{3}\left(\geq 99 \%\right.$, Sigma-Aldrich, USA), $\mathrm{BaCO}_{3}$ and $\mathrm{TiO}_{2}(99.8 \%$, Aldrich Chemistry, USA) were mixed, milled and dried using the same procedures presented above. The dried powder was calcined at $1350{ }^{\circ} \mathrm{C}$ for 2 hours with the same ramp rate in the same furnace as for the $\mathrm{BaZrO}_{3}$. Because the decomposition of $\mathrm{BaCO}_{3}$ and $\mathrm{CaCO}_{3}$ produces $\mathrm{CO}_{2}$, all the powder mixtures were calcined in open $\mathrm{Al}_{2} \mathrm{O}_{3}$ crucibles. The calcined powders were re-milled for 12 hours and dried using the same processes presented above, followed by sieving through a 200 $\mu \mathrm{m}$ sieve mesh. Green bodies were shaped by uniaxial single-ended dry pressing at $62 \mathrm{MPa}$ in a 
stainless steel die with $14.5 \mathrm{~mm}$ diameter. Before pressing, the powder was mixed with $8.8 \mathrm{wt} . \%$ of binder (3.3 wt.\% polyvinyl alcohol dissolved in deionized water). The binder in the green bodies was burnt off at $500{ }^{\circ} \mathrm{C}$ for 10 hours with a slow ramping rate of $1{ }^{\circ} \mathrm{C} \mathrm{min}{ }^{-1}$. The green bodies were sintered at $1450{ }^{\circ} \mathrm{C}$ with a $5{ }^{\circ} \mathrm{C} \min ^{-1}$ ramp rate for 4 hours on a $\mathrm{ZrO}_{2}$ substrate in air and in the same muffle furnace presented above. The surfaces of the sintered samples were polished on a P1200 silicon carbide abrasive paper (Eco-Wet, KWH Mirka Ltd., Finland) using water as the coolant. The polished sample surfaces were coated with Ag paste (DT 1402, Heraeus, Germany) to form electrodes through screen printing and subsequent firing $\left(600{ }^{\circ} \mathrm{C}, 20 \mathrm{mins}\right)$. The fabrication details of the KNBNNO samples can be found in reference [8].

Finally, the ferroelectric hysteresis loops (P-E loops) were measured with a ferroelectric test system (Precision LCII, Radiant Technologies, Inc., USA) at $1 \mathrm{~Hz}$. The temperature was controlled and maintained by dipping the samples in a beaker of silicone oil placed on a hot plate with temperature feedback (RCT Basic + ETS-D5, IKA, Germany).

\section{Validation and discussions}

The P-E loops of the BCZT measured at different temperatures and simulated according to the abovepresented model are shown in Figure 2. The shapes as well as the saturation and remanent polarization values of the simulated and measured loops agreed with each other to a large extent. Figure 3 compares the saturation polarization values for the measured and simulated results shown in Figure

2. Errors smaller than $5 \%$ between the measurements and simulations were obtained for all the temperatures, indicating a good agreement and reliable prediction from the developed model. More obvious disagreement between the measurements and the simulations was found for the coercive electric field, as shown in Figure 4. However, in this case the simulation results were still reasonable, as they showed a trend gradually decreasing to zero from the ferroelectric $\left(<90{ }^{\circ} \mathrm{C}\right)$ to the paraelectric 
phase $\left(>90{ }^{\circ} \mathrm{C}[11,34]\right)$. The increased level of disagreement between the simulations and measurements shown in Figure 4 compared to that of Figure 3 are considered to be due to the accuracy of the measurements. In addition, it should be noted that approximately $90{ }^{\circ} \mathrm{C}$, which is obtained from permittivity-temperature dependence measurement, is typically considered as the Curie temperature of BCZT $[11,34]$. However, in the P-E loop measurement the coercive force was not zero at about $90{ }^{\circ} \mathrm{C}$.
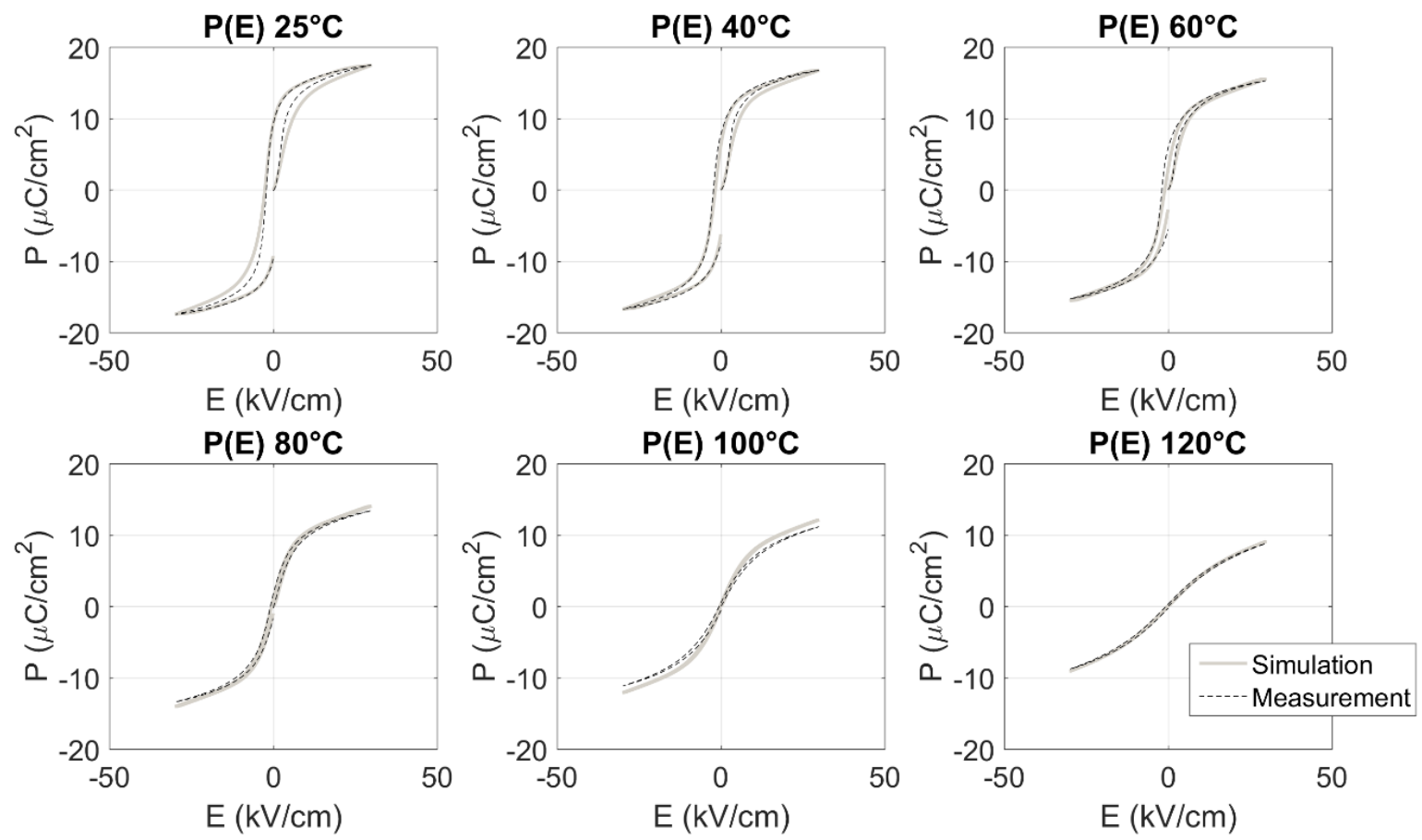

Figure 2. Dependence of polarization $(P)$ on electric field $(E)$ - P-E loops - for the BCZT measured at and simulated for different temperatures. 


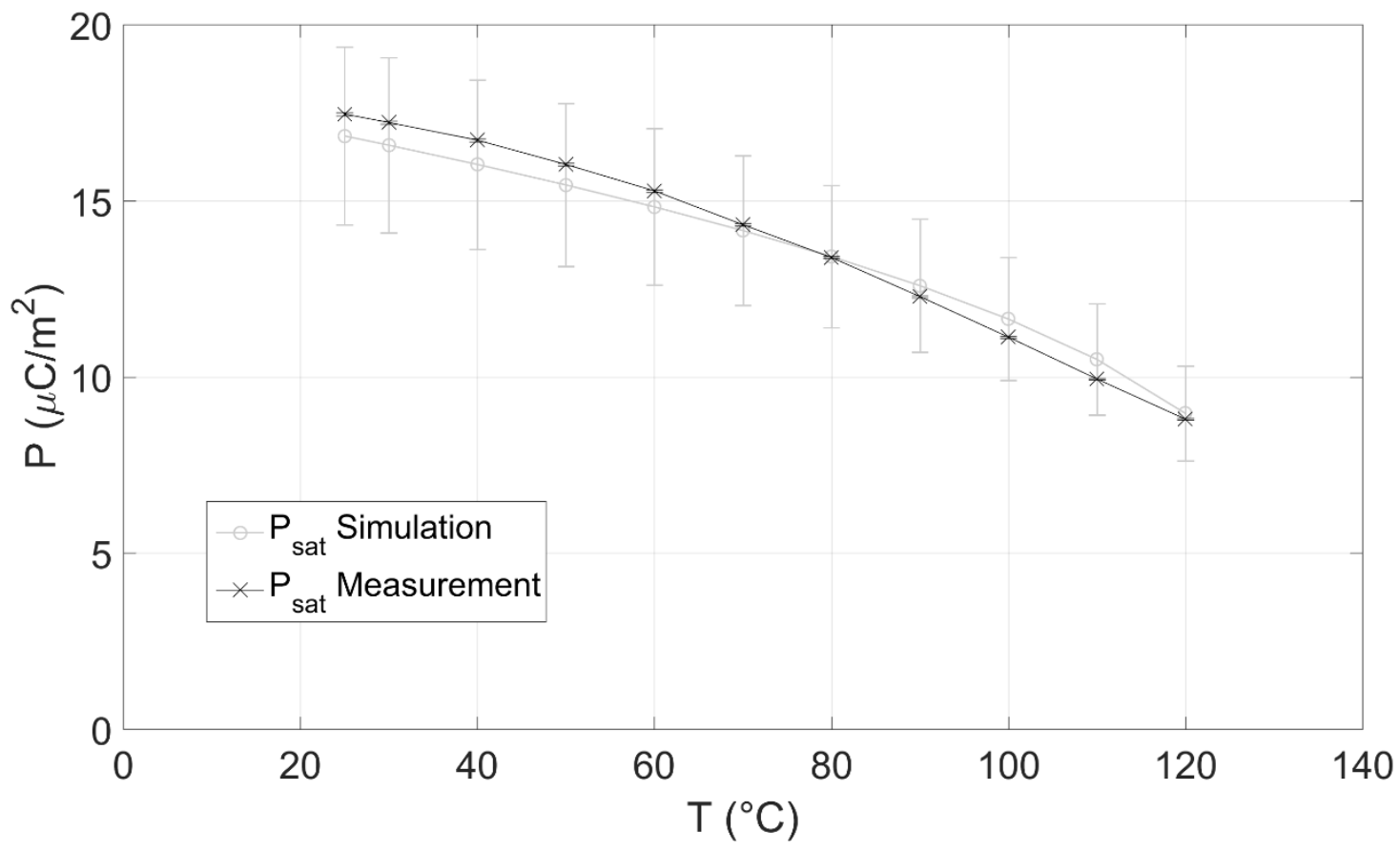

Figure 3. Dependence of saturation polarization $\left(P_{\text {sat }}\right)$ extracted from the P-E loops shown in Figure 2 on temperature $(T)$ for the BCZT with simulation and measurement.

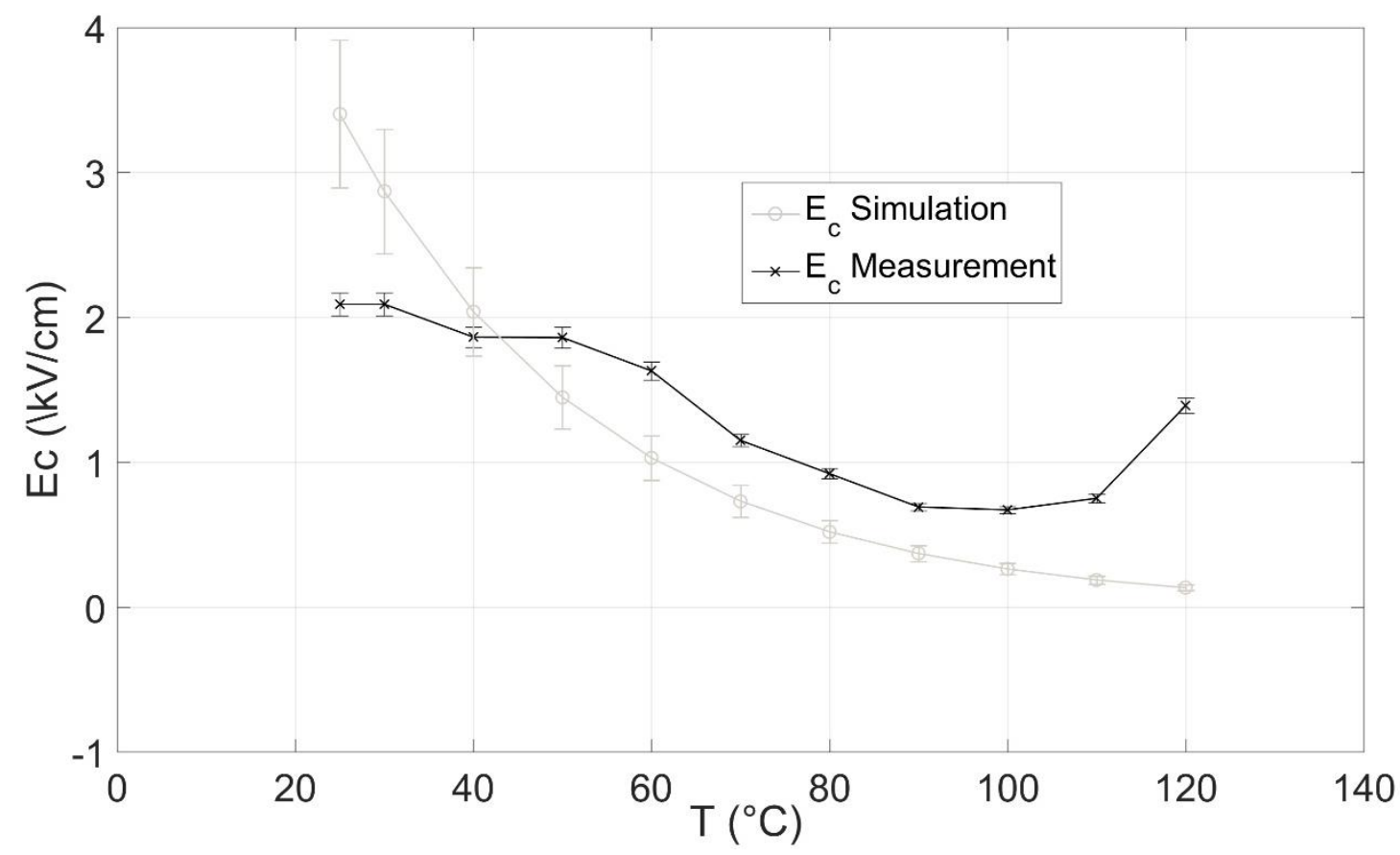

Figure 4. Dependence of coercive electric field $\left(E_{c}\right)$ extracted from the P-E loops shown in Figure 2 on temperature $(T)$ for the BCZT with simulation and measurement. 
In Figures 3 and 4, the error bars of the measured data were generated based on the corresponding standard deviations of the three measured samples in a batch. The error bars of the simulated data were added with the following consideration. The Jiles-Atherton original model in its ferroelectric version rely on five parameters. An optimization code based on the minimization of an error function was used to set these parameters, where the room temperature experimental results constituted the target of this optimization. For the temperature contribution added into the model as presented in this paper, the two additional parameters $\left(\beta\right.$ and $\left.T_{c}\right)$ might affect the results of the simulation. In this case, $\beta$ could be obtained from the optimization with the experimental data, while $T_{c}$ could be measured from the temperature-dependent ferroelectric behavior or deduced from the nature of the material reported in literature. In this work, better fittings were observed using a $T_{c}$ which was $10 \%$ lower than the measured one. This can be explained by the difference between the methods for $T_{c}$ measurements used in this work and in literature, as presented above. Therefore, such a $10 \%$ divergence was considered to be the error of the simulation data in Figures 3 and 4.

Figure 5 shows the P-E loops of the KNBNNO measured at different temperatures. As the differences between the loops were negligible, indicating a stable ferroelectric behavior over a wide range of temperatures, the simulation was only carried out at room temperature. Figure 6 compares the roomtemperature simulated and measured P-E loops (virgin loop polarization) for the KNBNNO. As in the case of BCZT, an excellent agreement between the simulation and measurement was achieved with nearly zero difference between the two was recognized, indicating an accurately developed model describing the ferroelectric behavior of both materials. The model is universal and can also be used for any other ferroelectric material. 


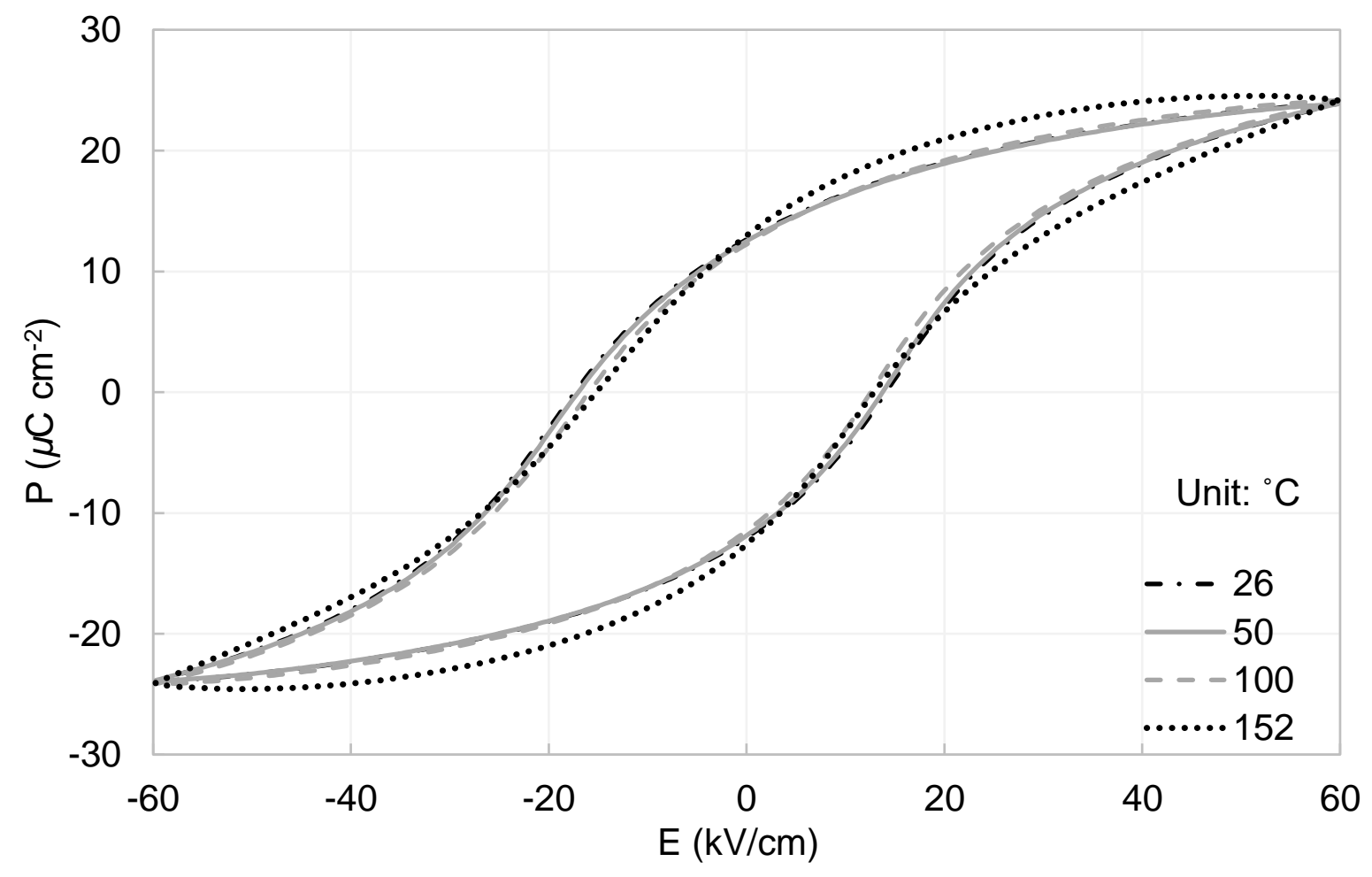

Figure 5. P-E loops of the KNBNNO (measured results) at $1 \mathrm{~Hz}$ and at different temperatures.

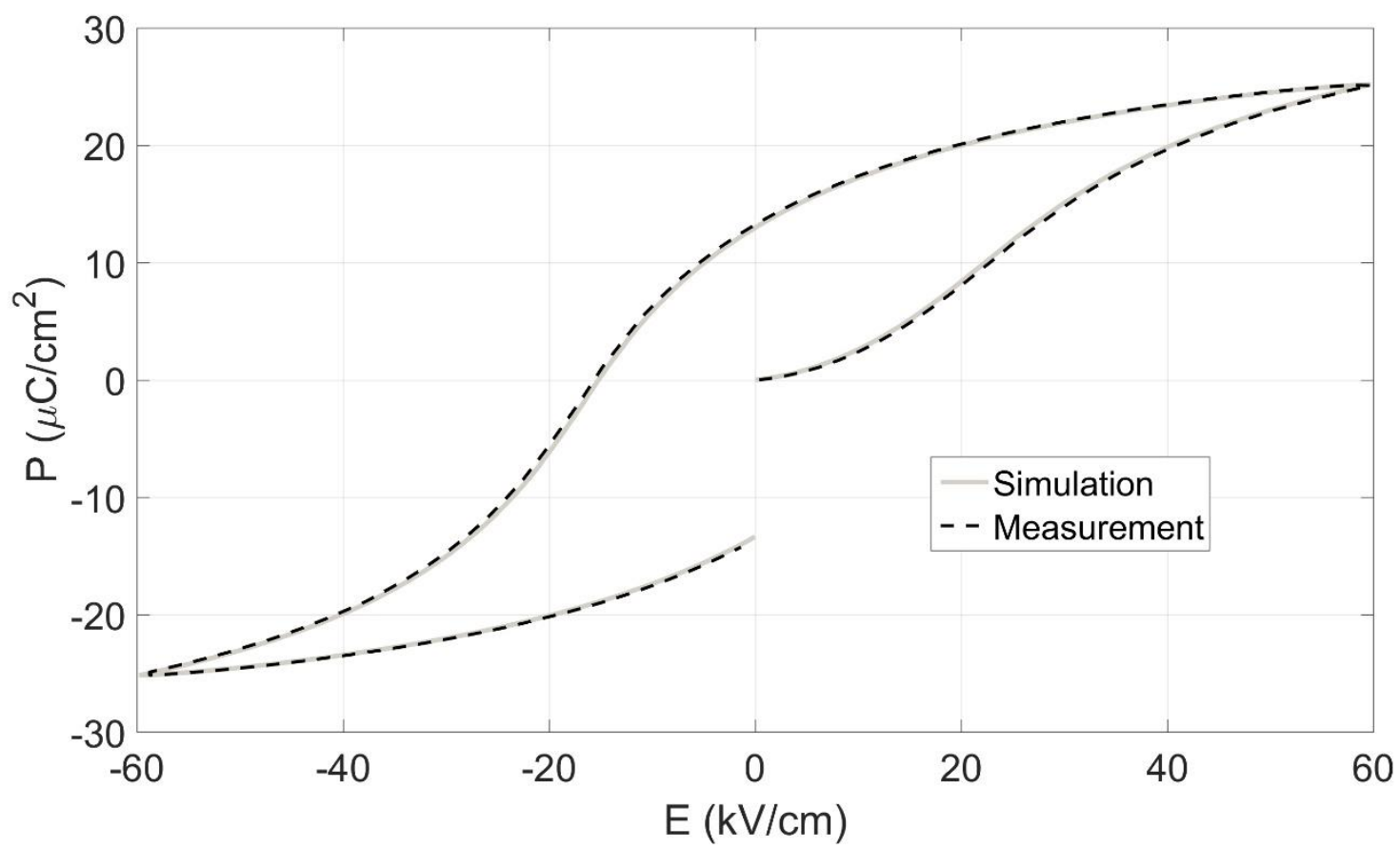

Figure 6. Measured and simulated virgin P-E loops of the KNBNNO at $1 \mathrm{~Hz}$ and at room temperature. 
As introduced with the model, the dynamic contribution was also considered. However, up to now the frequency range of the experimental setup is too limited to validate the frequency dependence. Improvements will be made in future works to reach high frequency levels, in order to validate the dynamic consideration for a widened frequency range. Relevant experimental results dealing with the frequency dependence of soft ferroelectrics are available in references [21-25].

\section{Conclusions}

A temperature-dependent model for ferroelectric behavior has been developed by modifying the original J-A model created for ferromagnetic materials. Dynamic and temperature contributions have been considered in the revised model to cover a wider frequency band and temperature variations, respectively. Two ferroelectric ceramic materials, BCZT and KNBNNO, have been fabricated and used for the validation of the model. Good agreements between the simulations and measurements have been achieved for both materials, especially at room temperature (ferroelectric phase) and at temperatures higher than the Curie temperature (paraelectric phase). The developed model is universal and can also be used to predict the P-E loops at different temperatures for any other ferroelectric material. The P-E loops define the spontaneous and remanent polarizations which affect the ferroelectric, piezoelectric, pyroelectric and multi-functional properties to a large extent. Therefore, being able to precisely simulate the loops using this developed model with the consideration of temperature and frequency enables a better understanding and optimization of a wide range of ferroelectric materials.

\section{Acknowledgements}


This work received funding from the European Union's Horizon 2020 research and innovation program under the Marie Sklodowska-Curie grant agreement number 705437. Author J.J. acknowledges the funding of the Academy of Finland (project numbers 267573, 273663 and 298409).

\section{References}

[1] Bowen C R, Kim H A, Weaver P M and Dunn S 2014 Piezoelectric and ferroelectric materials and structures for energy harvesting applications Energy Environ.Sci. 7 25-44

[2] Bowen C R, Taylor J, LeBoulbar E, Zabek D, Chauhan A and Vaish R 2014 Pyroelectric materials and devices for energy harvesting applications Energy Environ.Sci. 7 3836-56

[3] Damjanovic D 1998 Ferroelectric, dielectric and piezoelectric properties of ferroelectric thin films and ceramics Rep.Prog.Phys. 61 1267-324

[4] Bai Y, Jantunen H and Juuti J 2018 Energy Harvesting Research: The Road from Single-source to Multi-source Adv Mater. DOI: 10.1002/adma.201707271

[5] Roedel J, Jo W, Seifert K T P, Anton E, Granzow T and Damjanovic D 2009 Perspective on the Development of Lead-free Piezoceramics J Am Ceram Soc. 92 1153-77

[6] Bowen C R, Taylor J, Le Boulbar E, Zabek D and Topolov V Y 2015 A modified figure of merit for pyroelectric energy harvesting Mater Lett. 138 243-6

[7] Bai Y, Siponkoski T, Perantie J, Jantunen H and Juuti J 2017 Ferroelectric, pyroelectric, and piezoelectric properties of a photovoltaic perovskite oxide Appl.Phys.Lett. 110063903

[8] Bai Y, Tofel P, Palosaari J, Jantunen H and Juuti J 2017 A Game Changer: A Multifunctional Perovskite Exhibiting Giant Ferroelectricity and Narrow Bandgap with Potential Application in a Truly Monolithic Multienergy Harvester or Sensor Adv Mater. 291700767

[9] Grinberg I, West D V, Torres M, Gou G, Stein D M, Wu L, Chen G, Gallo E M, Akbashev A R, Davies P K, Spanier J E and Rappe A M 2013 Perovskite oxides for visible-light-absorbing ferroelectric and photovoltaic materials Nature. $\mathbf{5 0 3} 509$

[10] Paillard C, Bai X, Infante I C, Guennou M, Geneste G, Alexe M, Kreisel J and Dkhil B 2016 Photovoltaics with Ferroelectrics: Current Status and Beyond Adv Mater. 28 5153-68

[11] Bai Y, Matousek A, Tofel P, Bijalwan V, Nan B, Hughes H and Button T W 2015 $(\mathrm{Ba}, \mathrm{Ca})(\mathrm{Zr}, \mathrm{Ti}) \mathrm{O}-3$ lead-free piezoelectric ceramics-The critical role of processing on properties J.Eur.Ceram.Soc. 35 3445-56 
[12] Bai Y, Vats G, Seidel J, Jantunen H and Juuti J 2018 Boosting photovoltaic output of ferroelectric ceramics by opto-electric control of domains Adv Mater. Submitted work under revision

[13] Preisach F 1935 Über die magnetische Nachwirkung 94277

[14] Mayergoyz I 1986 Mathematical-Models of Hysteresis Phys.Rev.Lett. 56 1518-21

[15] Jiles D and Atherton D 1984 Theory of Ferromagnetic Hysteresis J.Appl.Phys. 55 2115-20

[16] Della Torre E 1998 Hysteresis modeling Compel-Int.J.Comp.Math.Electr.Electron.Eng. 17682

[17] Dellatorre E and Vajda F 1994 Parameter-Identification of the Complete-Moving-Hysteresis Model using Major Loop Data IEEE Trans.Magn. 30 4987-5000

[18] Zhang B, Gupta B, Ducharne B, Sebald G and Uchimoto T 2018 Preisach's model extended with dynamic fractional derivation contribution54 6100204

[19] Smith R and Hom C 1999 Domain wall theory for ferroelectric hysteresis J Intell Mater Syst Struct. 10 195-213

[20] Leite J, Sadowski N, Kuo-Nng P, Batistela N and Bastos J 2003 The inverse Jiles-Atherton model parameters identification IEEE Trans.Magn. 39 1397-400

[21] Guyomar D, Ducharne B and Sebald G 2008 Time fractional derivatives for voltage creep in ferroelectric materials: theory and experiment J.Phys.D-Appl.Phys. $\mathbf{4 1} 125410$

[22] Guyomar D, Ducharne B and Sebald G 2007 Dynamical hysteresis model of ferroelectric ceramics under electric field using fractional derivatives J.Phys.D-Appl.Phys. 40 6048-54

[23] Guyomar D, Ducharne B, Sebald G and Audiger D 2009 Fractional Derivative Operators for Modeling the Dynamic Polarization Behavior as a Function of Frequency and Electric Field Amplitude IEEE Trans.Ultrason.Ferroelectr.Freq.Control. 56 437-43

[24] Guyomar D, Ducharne B and Sebald G 2010 High frequency bandwidth polarization and strain control using a fractional derivative inverse model 19045010

[25] Guyomar D, Ducharne B and Sebald G 2010 The use of fractional derivation in modeling ferroelectric dynamic hysteresis behavior over large frequency bandwidth J.Appl.Phys. 107 114108

[26] Grünwald A K 1867 Ueber begrenzte derivationen und deren Anwendung 44180

[27] Riemann B 1892 Besammelte Werke

[28] Liouville J 1983 Mémoire sur le calcul des différentielles à indices quelconques 1371

[29] Ducharne B, Sebald G, Guyomar D and Litak G 2015 Dynamics of magnetic field penetration into soft ferromagnets J.Appl.Phys. 117243907 
[30] Raghunathan A, Melikhov Y, Snyder J E and Jiles D C 2009 Modeling the Temperature Dependence of Hysteresis Based on Jiles-Atherton Theory IEEE Trans.Magn. 45 3954-7

[31] Arrott A and Noakes J E 1967 Approximate equation of state for nickel near its critical temperature 19786

[32] Leite J, Avila S, Batistela N, Carpes W, Sadowski N, Kuo-Peng P and Bastos J 2004 Real coded genetic algorithm for Jiles-Atherton model parameters identification IEEE Trans.Magn. 40 888-91

[33] Marion R, Scorretti R, Siauve N, Raulet M and Kraehenbuehl L 2008 Identification of JilesAtherton model parameters using particle swarm optimization IEEE Trans.Magn. 44 894-7

[34] Liu W and Ren X 2009 Large Piezoelectric Effect in Pb-Free Ceramics Phys.Rev.Lett. 103 257602 